\title{
Three-grating monolithic phase-mask for the single-order writing of large-period gratings
}

\author{
Yannick Bourgin \\ yannick.bourgin@univ-st-etienne.fr \\ Ismo Vartiainen \\ Yves Jourlin \\ Markku Kuittinen \\ Frédéric Celle \\ Svetlen Tonchev

\section{Olivier Parriaux}

\section{Tapio Niemi}

\author{
Université de Lyon, Laboratoire Hubert Curien, UMR CNRS 5516, 18 rue du Pr. B. Lauras, 42000 \\ Saint-Etienne, France \\ University of Eastern Finland, Department of Physics and Mathematics, P.O Box 111, 80101 Joensuu, \\ Finland \\ Université de Lyon, Laboratoire Hubert Curien, UMR CNRS 5516, 18 rue du Pr. B. Lauras, 42000 \\ Saint-Etienne, France \\ University of Eastern Finland, Department of Physics and Mathematics, P.O Box 111, 80101 Joensuu, \\ Finland \\ Université de Lyon, Laboratoire Hubert Curien, UMR CNRS 5516, 18 rue du Pr. B. Lauras, 42000 \\ Saint-Etienne, France \\ Université de Lyon, Laboratoire Hubert Curien, UMR CNRS 5516, 18 rue du Pr. B. Lauras, 42000 \\ Saint-Etienne, France \\ Université de Lyon, Laboratoire Hubert Curien, UMR CNRS 5516, 18 rue du Pr. B. Lauras, 42000 \\ Saint-Etienne, France \\ Tampere University of Technology, P.O. Box 527, 33101 Tampere, Finland
}

An optimized achromatic high-efficiency monolithic phase mask is presented whose principle was demonstrated and described in reference [1]. The mask comprises three submicron period diffraction gratings at a single substrate side that create a purely single spatial frequency interferogram of large period. The optical scheme is that of an integrated Mach-Zehnder interferometer where all light circulation functions are performed by diffraction gratings. The paper describes the operation principle of the phase mask, the fabrication process, and its utilization in a write-on-the-fly scheme for the writing of a long, $2 \mu \mathrm{m}$-period grating. [D0I: 10.2971/jeos.2011.11016s]

Keywords: micro-optics, diffraction grating, optical lithography, nanostructure fabrication

\section{INTRODUCTION}

Phase masks have long been used for the fabrication of Fibre Bragg Gratings (FBGs) [2]-[4]. They offer the notable advantage over the more classical technique of creating an interferogram in a Mach-Zehnder interference scheme [5] that the period and the location of the created interferogram only depend on the period and location of the phase mask grating. The price to pay is the impossibility of adjusting the period of the interferogram which is imprinted in the phase mask corrugation. This is however a minor drawback in the writing of long gratings of constant period [6], and in the writing of a number of identical gratings. Another penalty is the restriction of the range of periods which can possibly be printed: the generated interferogram only has a single spatial frequency in the operation regime where the $+1^{\text {st }}$ and $-1^{\text {st }}$ orders at least and at most can propagate. Somewhat paradoxically, it is therefore more difficult to write a supmicron than a submicron period grating with a phase mask since the sensitivity spectrum of most photoresists is below $500 \mathrm{~nm}$ wavelength. Micrometer-scale period gratings are however needed as in IR spectroscopy and in grating scales for displacement sensors for instance where phase mask printing would be very advantageous industrially when associated with a write on the fly strategy [6].

The present paper describes an alternative phase mask structure permitting the generation of a high contrast, single spatial frequency interferogram of arbitrarily large period. It is an integrated monolithic Mach-Zehnder scheme operating in the spatial frequency domain similarly to an heterodyne frequency scheme: the phase mask comprises a central beam splitting grating, and two lateral grating zones at either side of the central corrugation and playing the role of the mirrors of the Mach-Zehnder interferometer. The spatial frequency of the generated interferogram is equal to twice the difference between the spatial frequencies of the central and lateral gratings. After the functionality of this dual-grating phase mask has been demonstrated with a laboratory prototype based on a simple photoresist technology [1], we will report here on its optimization and implementation in hard materials by means of e-beam writing and reactive ion etching. 


\section{PHASE MASK PRINCIPLE}

The device described here is a monolithic phase mask composed of 3 diffraction gratings: a wave splitting grating of period $\Lambda_{1}$ with two gratings of period $\Lambda_{2}$ aside the latter. The functionality of such monolithic phase-mask was demonstrated by using photoresist gratings defined at the incidence side of a thick glass substrate [1]. Despite its poor efficiency, the principle was demonstrated of splitting an incident beam with a transmission grating $G_{1}$ of period $\Lambda_{1}$ directing its + and $-1^{\text {st }}$ diffracted orders to two reflection gratings $\mathrm{G}_{2}$ of period $\Lambda_{2}$ that redirect both incoming beams via their $-1^{\text {st }}$ diffraction order below the substrate where they overlap and interfere. In the present paper the principle is the same even though the configuration is different. An exposure laser beam enters the substrate through the air $/ \mathrm{SiO}_{2}$ interface and impinges onto grating $\mathrm{G}_{1}$ of period $\Lambda_{1}$ located on the opposite side of the substrate. This grating generates the + and $-1^{\text {st }}$ orders in reflection. The line/space and the aspect ratio of this reflection grating are chosen to essentially cancel the $0^{\text {th }}$ reflected order. The diffracted orders propagate upwards in the substrate and are reflected at the opposite $\mathrm{SiO}_{2} /$ Air interface under Total Internal Reflection. Then they impinge onto two diffraction gratings $\mathrm{G}_{2}$ of period $\Lambda_{2}$ located at each side of $\mathrm{G}_{1}$. The function of gratings $G_{2}$ is to redirect the beams coming from $G_{1}$ outside the substrate using the $-1^{\text {st }}$ transmitted order. The parameters of gratings $G_{1}$ and $G_{2}$ are chosen so as to optimize the overall diffraction efficiency. Their design will be described hereunder. Figure 1 is a diagram of the monolithic phase-mask element.

Eq. (1) relates the grating periods $\Lambda_{1}$ and $\Lambda_{2}$ of gratings $G_{1}$ and $G_{2}$ and the period $p$ of the fringes pattern generated in the overlap zones of the two beams. This phase mask is achromatic, i.e. the interferogram period $p$ only depends on $\Lambda_{1}$ and $\Lambda_{2}$.

$$
\frac{1}{p}=2 *\left(\frac{1}{\Lambda_{2}}-\frac{1}{\Lambda_{1}}\right)
$$

\section{MODELLING}

The objective of the work presented here was to find out and to design the optimum configuration permitting to print a grating of $2 \mu \mathrm{m}$ period. Such period is not printable by means of a standard phase mask because of the complexity of the multi-order generated interferogram and it was shown to be achievable by means of a Mach-Zehnder interferometer configuration [1]. Periods $\Lambda_{1}=420 \mathrm{~nm}$ and $\Lambda_{2}=380 \mathrm{~nm}$ do generate an interferogram of $2 \mu \mathrm{m}$ period according to Eq. (1), furthermore, $G_{1}$ gives rise at the $442 \mathrm{~nm}$ wavelength of an $\mathrm{HeCd}$ laser to diffracted beams experiencing total internal reflection at the opposite substrate side. A code based on the true-mode method [7] is used to find the parameters optimizing the diffraction efficiencies. Grating 1 of period $\Lambda_{1}$ will be etched in the fused silica substrate and coated with aluminium. The code is used to optimized the width and the

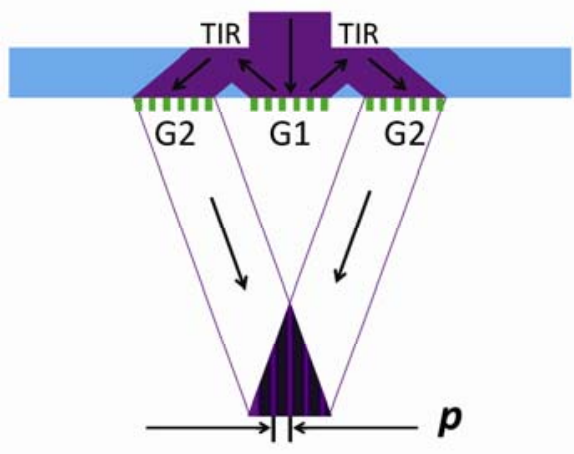

FIG. 1 Monolithic phase mask and related beam path.

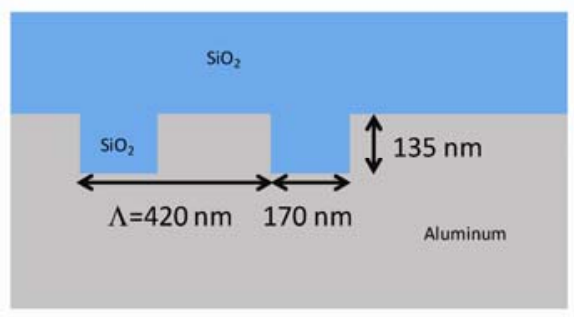

FIC. 2 Sketch of the binary corrugation of grating $G_{1}$ etched in the fused silica substrate and filled with aluminium.
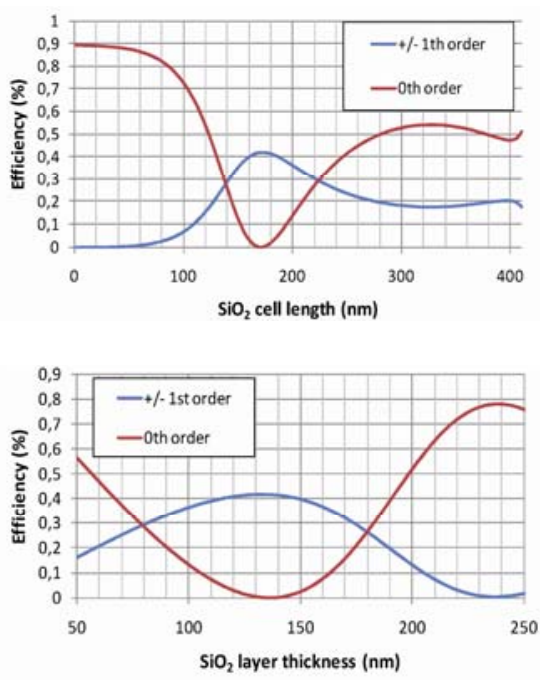

FIG. 3 Power efficiency of the diffracted orders of $\mathrm{G}_{1}$ versus the $\mathrm{SiO}_{2}$ line width. Down: The same versus the $\mathrm{SiO}_{2}$ line height.

depth of the fused silica lines to have the maximum power distributed in the + and $-1^{\text {st }}$ orders and minimizing the $0^{\text {th }}$ reflected order. Figure 2 is a schematic sketch of the optimized structure. Figure 3 shows the power efficiencies of the $0^{\text {th }}$ reflected orders and the $+/-1^{\text {st }}$ reflected orders. Figure 3 top gives the power efficiency versus the line width and the curves at the bottom show the same versus the $\mathrm{SiO}_{2}$ etched depth.

The curves obtained by the modelling code show that for a $\mathrm{SiO}_{2}$ line width of $170 \mathrm{~nm}$ the efficiency of the + and $-1^{\text {st }}$ re- 
flected diffraction orders has a maximum $(41.67 \%$ of the incident power) while the $0^{\text {th }}$ reflected order admits a minimum $(0.02 \%)$. Fixing now the $\mathrm{SiO}_{2}$ line width at $170 \mathrm{~nm}$, Figure 3 top shows the tolerance on the etching depth with a maximum at $135 \mathrm{~nm}$. The optimized parameters of $\mathrm{G}_{1}$ are summarized in Table 1.

\begin{tabular}{|c|c|c|}
\hline Grating 1 & \multicolumn{2}{|c|}{ period $=420 \mathrm{~nm}$} \\
\hline incident angle & \multicolumn{2}{|c|}{$0^{\circ}$} \\
\hline $\mathrm{SiO}_{2}$ layer thickness & \multicolumn{2}{|c|}{$135 \mathrm{~nm}$} \\
\hline $\mathrm{SiO}_{2}$ line width & \multicolumn{2}{|c|}{$170 \mathrm{~nm}$} \\
\hline Order & angle & efficiency \\
\hline Reflected 0 & $0^{\circ}$ & $0.02 \%$ \\
\hline Reflected -/+1 & $45.831^{\circ}$ & $41.67 \%$ \\
\hline Transmitted 0 & $0^{\circ}$ & $0.00 \%$ \\
\hline
\end{tabular}

TABLE 1 Modelling parameters used for $G_{1}$ and expected orders efficiencies.

Transmission gratings $G_{2}$ have been designed by using an incidence angle on the grating from the fused quartz substrate side equal to $45.8^{\circ}$ according to the modelling result of $G_{1} \cdot G_{2}$ is a grating of period $380 \mathrm{~nm}$ with grating lines etched all through a high refractive index material $\left(\mathrm{Si}_{3} \mathrm{~N}_{4}, n\right.$ $=2.07, k=0.006 @ \lambda=441.6 \mathrm{~nm}$ ) with air under and between the silicon nitride lines. Figure 4 is a sketch of grating $\mathrm{G}_{2}$.

The results of the modelling are presented in Figure 5, at the top the curves represent the power efficiency of the $-1^{\text {st }}$ (transmitted -1 in Table 2) transmitted diffracted order versus the $\mathrm{Si}_{3} \mathrm{~N}_{4}$ line width, and at the bottom versus the thickness of the $\mathrm{Si}_{3} \mathrm{~N}_{4}$ layer.

\begin{tabular}{|c|c|c|}
\hline Grating 2 & \multicolumn{2}{|c|}{ period $=380 \mathrm{~nm}$} \\
\hline incident angle & \multicolumn{2}{|c|}{$45.831^{\circ}$} \\
\hline $\begin{array}{c}\mathrm{Si}_{3} \mathrm{~N}_{4} \text { layer thick- } \\
\text { ness }\end{array}$ & \multicolumn{2}{|c|}{$210 \mathrm{~nm}$} \\
\hline $\mathrm{Si}_{3} \mathrm{~N}_{4}$ line width & \multicolumn{2}{|c|}{$105 \mathrm{~nm}$} \\
\hline Order & angle & efficiency \\
\hline Reflected -2 & $-60.262^{\circ}$ & $2.92 \%$ \\
\hline Reflected -1 & $-4.33^{\circ}$ & $5.08 \%$ \\
\hline Reflected 0 & $45.831^{\circ}$ & $1.21 \%$ \\
\hline Transmitted -1 & $-6.3529^{\circ}$ & $87.85 \%$ \\
\hline
\end{tabular}

TABLE 2 Modelling parameters used for $G_{2}$ and expected orders efficiencies.

The curves of figure 5 show that the optimal parameters for $\mathrm{G}_{2}$ are: gratings lines with a depth of $210 \mathrm{~nm}$ all through the

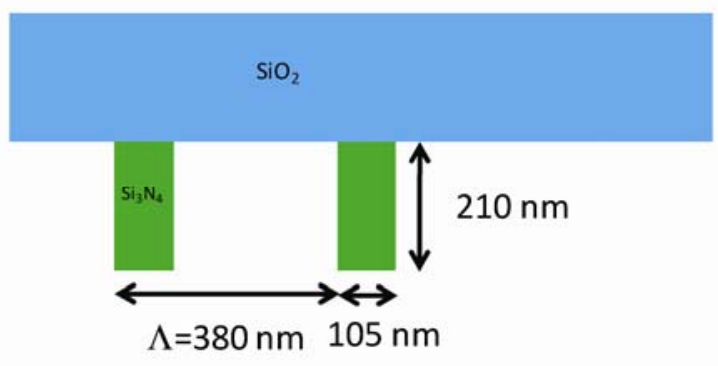

FIG. 4 Sketch of grating $C_{2}$ etched all through a silicon nitride layer.
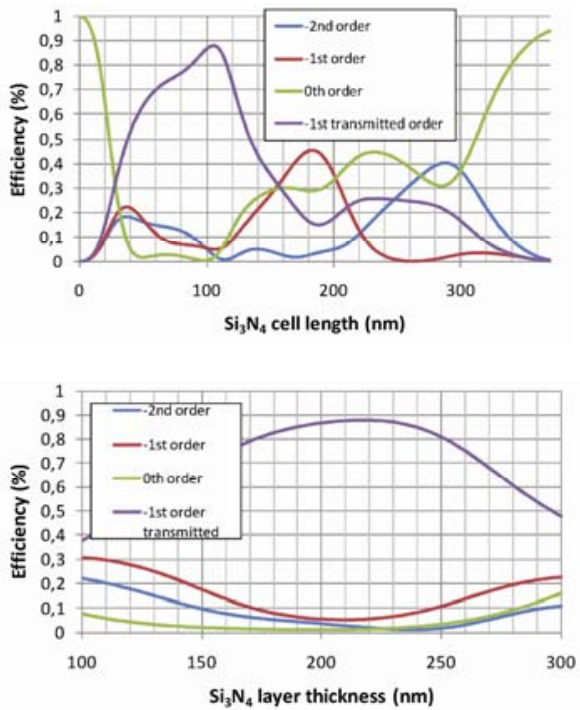

FIG. 5 Power efficiency of the diffracted orders of $\mathrm{G}_{2}$ versus the $\mathrm{Si}_{3} \mathrm{~N}_{4}$ line width. Down: The same versus the $\mathrm{Si}_{3} \mathrm{~N}_{4}$ line height.

$\mathrm{Si}_{3} \mathrm{~N}_{4}$ layer with a line/space ratio of $105 / 380=0.28$. Table 2 summarizes the parameters and gives the efficiency distribution in each diffracted orders generated by $\mathrm{G} 2$.

The expected efficiency of the phase mask is given by the product of the efficiencies of the $-1^{\text {st }}$ reflected order of $\mathrm{G}_{1}$ by that of the $-1^{\text {st }}$ transmitted order of $G_{2}$ multiplied by 2 . With the optimal parameters the theoretical efficiency is: $0.417^{*} 0.879 * 2=73.3 \%$.

\section{PHASE MASK FABRICATION}

The most important feature in the dual-grating phase mask is the parallelism between all grating lines to prevent any Moiré effect in the interferogram. This is the reason for writing all three grating zones at the same substrate side to prevent any tilt resulting from unloading and reloading the substrate on the e-beam chuck. A silicon nitride layer was deposited by PECVD (Plasma Enhanced Chemical Vapour Deposition) on a thick (3 mm) fused silica substrate. The corrugations of $G_{1}$ and $G_{2}$ are not identical; $G_{1}$ is reflective whereas $G_{2}$ is a transmission grating. Therefore the samples must be prepared before the e-beam writing. $G_{1}$ will be etched in the fused silica substrate while the gratings $G_{2}$ will be etched in 


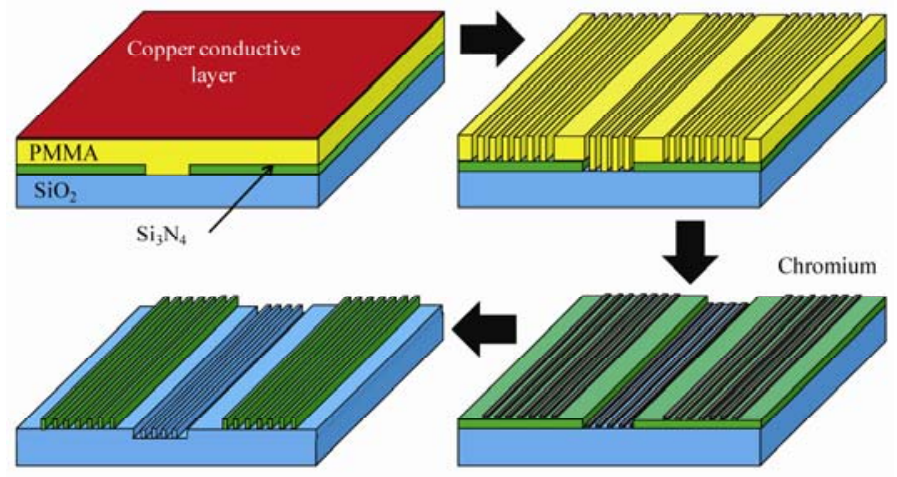

Fig. 6 Phase mask grating fabrication steps. Top left: e-beam resist and copper layer deposition. Top right: Grating pattern in the resist layer. Bottom right: Lift-off of the chromium etch-mask. Bottom left: Etching in $\mathrm{SiO}_{2}\left(\mathrm{G}_{1}\right)$ and $\mathrm{Si}_{3} \mathrm{~N}_{4}\left(\mathrm{G}_{2}\right)$.

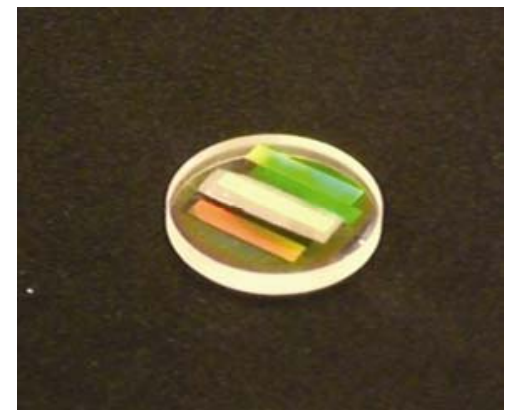

Fig. 7 Picture of a monolithic phase mask made on a 1 inch substrate. Gratings $G_{2}$ are visible at both sides of grating $\mathrm{G}_{1}$ covered by an aluminium layer.

the $\mathrm{Si}_{3} \mathrm{~N}_{4}$ layer. A window in the Silicon Nitride layer is first opened for $\mathrm{G}_{1}$ by using a RIE (Reactive Ion Etching) step down to the silica surface. A plastic foil is used to delimit the areas of the $\mathrm{SiO}_{2}$ window.

A layer of positive e-beam photoresist (AR-P 661 PMMA "PolyMethyl MethAcrylate"-based) is deposited by spin coating, and covered by a copper layer deposited by thermal evaporation to permit the evacuation of the electric charges. The grating lines are then written in the photoresist by a Vistec EBPG 5000+ electron beam nanowriter. The copper conductive layer is removed by wet etching, and the grating lines are revealed by using the appropriate development process. The lift-off technique is used to create the etch-mask for the RIE processes. A chromium layer $(25 \mathrm{~nm})$ is deposited by thermal evaporation at the bottom of the grooves and at the top of the photoresist layer. The sample is dipped in acetone for lifting off the chromium layer on the resist layer and keeping it on the other areas to be used as an etch-mask. The $\mathrm{Si}_{3} \mathrm{~N}_{4}$ layer is etched by $\mathrm{CHF}_{3}$-based RIE down to the silica surface (i.e. $185 \mathrm{~nm}$ ), a plastic foil protecting the $\mathrm{G}_{1}$ site. The same operation is performed for the etching of the $\mathrm{SiO}_{2}$ grating with the same etchant at $135 \mathrm{~nm}$ depth, the $G_{2}$ sites now being protected by a plastic foil. After the etching steps the chromium rests on top of the gratings is removed by an ammonium cerium (IV) nitrate and acetic acid based wet etching. The operations are summarized in Figure 6 .

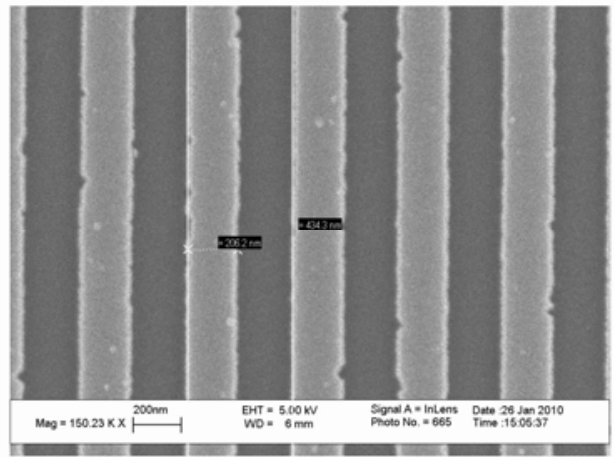

Fig. 8 SEM picture of grating $G_{1}$ before aluminium deposition

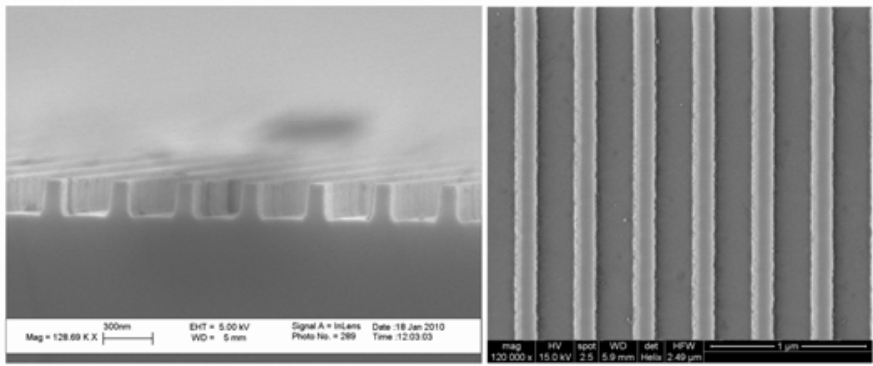

Fig. 9 SEM pictures of grating $G_{2}$. Left: side view. Right: top view.

Grating $G_{1}$ is then made reflective by aluminium evaporation, a plastic foil being used to protect the rest of the substrate. A high vacuum $\left(5^{*} 10^{-7} \mathrm{mbar}\right)$ and a sufficiently slow deposition rate $(2 \AA / s)$ are used to guarantee the correct filling of the grooves by aluminium. If the deposition speed is too high, the aluminium in the grooves grows in the form of aggregates. Figure 7 is a picture of the monolithic phase mask made on a $3 \mathrm{~mm}$-thick substrate (1 inch diameter) with grating line length of $15 \mathrm{~mm}$.

\section{CHARACTERIZATION}

The phase mask was characterized by means of SEM scans performed from the top and also from the edge after cleaving one of fabricated phase masks.

The SEM pictures of $G_{1}$ and $G_{2}$ show that the corrugations have close to the desired aspect ratio. Figure 9 left is a side picture of grating $G_{2}$ etched in the $\mathrm{Si}_{3} \mathrm{~N}_{4}$ layer. The shape of the grating lines is very close to a rectangle with the optimized parameters.

The phase masks were also characterized optically; the powers of the two output beams generated by the phase mask have been measured. The efficiency of the device is defined as the ratio between the power of the incident beam on $G_{1}$ and the sum of the output beams. The best sample shows an 


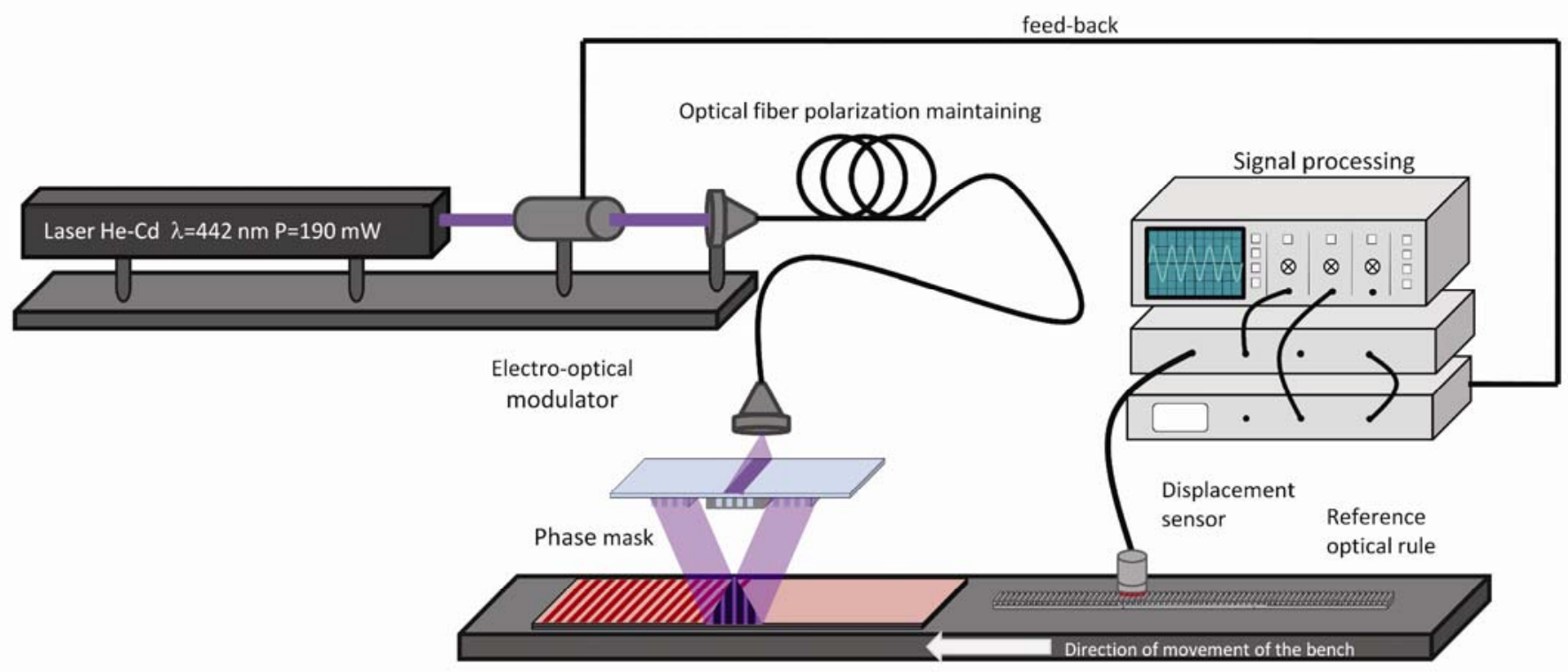

Fig. 10 Diagram of the "write on the fly" bench.

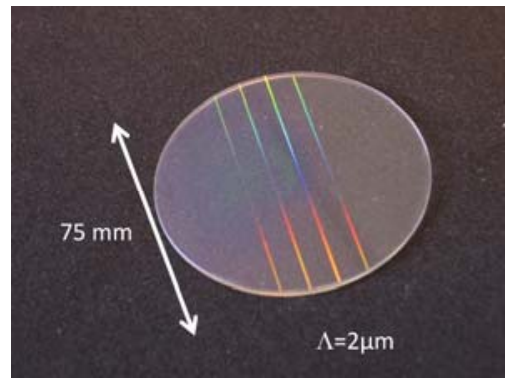

Fig. 11 Pictures of $2 \mu \mathrm{m}$ period grating tracks written with the "write on the fly" bench.

efficiency of $71 \%$, which is very close to the $73 \%$ expected by modelling the optimized gratings.

\section{LONG GRATING PRINTING}

The phase mask shown in Figure 7, and exhibiting the best efficiency was implemented on a "write on the fly" bench [6]. Figure 10 describes its operation: the principle is based on Scanning Beam Interference Lithography (SBIL). The phase mask is illuminated by a collimated laser beam (He-Cd laser, $\lambda=441.6 \mathrm{~nm}$ ) of $1.5 \mathrm{~mm}$ diameter under normal incidence. An interference pattern of $2 \mu \mathrm{m}$ period is created in the overlap volume of the two diffracted beams.

The lines of the interferogram are perpendicular to the displacement direction of the bench. A long substrate covered with photoresist (Shipley SPR 505) is placed on the translation bench, the distance between the phase mask and the substrate being adjusted so as to have the photoresist layer at about the middle of the beam overlap volume. The displacement of the stage is controlled by a grating displacement sensor of $1 \mu \mathrm{m}$ period. The $500 \mathrm{~nm}$ period sinusoidal electri- cal signal is TTL converted. An electro-optical modulator cuts the laser beam each time the substrate travels by half a period (i.e. $1 \mu \mathrm{m}$ ) and lets it through for a second period half to prevent a washing out of the printed latent grating.

Figure 11 is a picture of 4 diffraction gratings tracks of $2 \mu \mathrm{m}$ period. They have been written on 3 inch substrates at a speed of $800 \mu \mathrm{m} / \mathrm{s}$.

\section{CONCLUSION}

The proof of principle of a new monolithic dual-grating phase mask was made with the printing of a $2 \mu \mathrm{m}$ period grating using a $441.6 \mathrm{~nm}$ wavelength laser beam. The range of grating periods is easily controlled by the splitter and recombiner gratings. By changing the period of $\mathrm{G}_{2}$, periods between $250 \mathrm{~nm}$ and $2 \mu \mathrm{m}$ and over can be written with a pure single spatial frequency interferogram. The high efficiency obtained by using high index splitter and recombiner gratings to achieve more than $70 \%$ efficiency permits high writing speed dynamic exposure and reduced exposure time. By lengthening the grating lines of the monolithic phase mask up to the dimension which an e-beam nanowriter can write (5 inches for the Vistec used), large and long diffraction gratings of high spatial coherence can be written efficiently

\section{ACKNOWLEDGEMENTS}

The authors wish to thanks the FinNano-program of the Finnish Funding Agency for Technology and Innovation, for funding this project. We are very grateful to the photonics team of the Department of Physics and Mathematics of the University of Eastern Finland, Joensuu, for the technological support offered by the nano fabrication unit. 


\section{References}

[1] Y. Bourgin, S. Bakkali, Y. Jourlin, S. Tonchev, 0. Parriaux, "Monolithic double-grating phase mask for large-period highly coherent grating printing" Opt. Lett. 34, 3800-3802 (2009).

[2] M. Sumetsky, B.J. Eggleton, P.S. Westbrook, “Holographic methods for phase mask and fiber grating fabrication and characterization" Proc. SPIE 4941, 1-15 (2003).

[3] K.O. Hill, B. Malo, F. Bilodeau, D.C. Johnson and J. Albert, “Bragg gratings fabricated in monomode photosensitive optical fiber by UV exposure through a phase mask" Appl. Phys. Lett. 62, 10351038 (1993).

[4] P. E. Dyer, R.J. Farley and R. Giedl, "Analysis of grating formation with excimer laser irradiated phase masks" Opt. Commun. 115 , 327-334 (1995).
[5] G. C. Chen, P. T. Konkola, R. K. Heilmann and M. L. Schattenburg, "Nanomater-accurate grating fabrication with scanning beam interference lithography" Proc. SPIE 4936, 126-134 (2003).

[6] E. Gamet, Y. Jourlin, S. Pelissier, R. Min, S. Reynaud, C. Veillas, J. -C. Pommier and 0. Parriaux, "Flying phase mask for the printing of long submicron-period stitchingless gratings" Microelectron. Eng. 83, 734-737 (2006).

[7] http://www.mcgrating.com/index.php?page_id=1 (last checked February 2011) 